\title{
Left invertibility of discrete systems with finite inputs and quantized output
}

\author{
Nevio Dubbini, Benedetto Piccoli, Antonio Bicchi
}

\begin{abstract}
The aim of this paper is to address left invertibility for dynamical systems with inputs and outputs in discrete sets. We study systems that evolve in discrete time within a continuous state-space. Quantized outputs are generated by the system according to a given partition of the state-space, while inputs are arbitrary sequences of symbols in a finite alphabet, which are associated to specific actions on the system. We restrict to the case of contractive dynamics for fixed inputs. The problem of left invertibility, i.e. recovering an unknown input sequence from the knowledge of the corresponding output string, is addressed using the theory of Iterated Function Systems (IFS), a tool developed for the study of fractals. We show how the IFS naturally associated to a system and the geometric properties of its attractor are linked to the left invertibility property of the system. Our main results are a necessary and sufficient condition for a given system to be left invertible with probability one on the space of inputs (i.e. for almost all input sequences), and necessary and sufficient conditions for left invertibility and uniform left invertibility under some weak additional hypotheses. A few examples are presented to illustrate the application of the proposed method.
\end{abstract}

\section{INTRODUCTION}

Invertibility of dynamical systems is a fundamental problem of systems theory, and is distinguished in two aspects: right invertibility, which is concerned with surjectivity of the I/O map; and left invertibility, corresponding to injectivity of the map. While right inversion allows to find inputs and initial conditions which can produce a given output, left invertibility deals with the possibility of recovering unknown inputs from the knowledge of the outputs.

Invertibility problems are of interest in applications like fault detection in Supervisory Control and data Acquisition (SCADA) systems, system identification, and cryptography ([15], [9]). Invertibility of linear systems is a well understood problem, pioneered by [4], and then considered with algebraic approaches (see e.g. [25]), frequency domain techniques ([16], [17]), and geometric tools (cf. [19]). More recent work has addressed the invertibility of nonlinear systems ([23]). Right-invertibility is studied with differential geometry methods for instance in [20] and [22] for classes

Nevio Dubbini is with "Università di Pisa, Dipartimento di Matematica Leonida Tonelli”, Largo Bruno Pontecorvo 5, 56127, Pisa, Italy. dubbini@mail.dm. unipi.it

Benedetto Piccoli is with "Istituto per le Applicazioni del Calcolo Mauro Picone, Consiglio Nazionale delle Ricerche", Viale del Policlinico 137, I00161 Roma, Italy. b.piccolidiac.cnr.it

Antonio Bicchi is with "Università di Pisa, Centro Interdipartimentale di Ricerca E. Piaggio, via Diotisalvi 2, 56100 Pisa, Italy. bicchieing . unipi.it of smooth nonlinear systems. In [29], the left invertibility problem for a switched system is discussed.

This paper deals with left invertibility of a class of discrete-time nonlinear dynamical systems in a continuous state-space with inputs and outputs in finite sets. In particular, we consider the case that inputs are arbitrary sequences of symbols in a finite alphabet, each symbol being associated to a specific action on the system. Information available on the system is represented by sequences of output values in a discrete set. Such outputs are obtained by quantization, i.e. are generated by the system evolution according to a given partition of the state-space.

Quantized control systems have been attracting increasing attention of the control community in recent years (see [7], [27], [21] and references therein). The mathematical operation of quantization and the possibility of considering only finite inputs occurs in many communication and control systems. Finite inputs arise because of the intrinsic nature of the actuator, or anyway wherever the system operates under a logical supervisor. On the other hand, output quantization may occur because of the digital nature of the sensor, or if data need to be digitally transmitted. Most recently, the attention to quantization has been stimulated by the growing number of application involving "networked" control systems, which are systems interconnected through channels of limited capacity (see e.g. [28], [2]).

The problem considered in this paper is that of determining whether a given quantized system is left invertible. To this purpose, we first define the properties of distinguishability and uniform distinguishability of two input sequences. Loosely speaking, two input sequences are distinguishable if they generate two output strings that differ from each other on a finite time horizon. The main tool used in the paper is the theory of Iterated Function Systems (IFS). IFS have been already used as a model in different fields ([5], [24]). One can construct a natural map in the space of compact subsets of the euclidean space, simply by mapping a set in the union of the images of all contractions forming the IFS. The resulting dynamical systems has a unique attractor, which is also a compact set. Using recent results, we can determine the properties of the original control systems in terms of such compact attractor. More precisely, for every finite subset $C$ of the finite input alphabet, there exists an attractor $\mathscr{A}_{C}$. If all attractors are not inside a particular "diagonal" set, then almost every couple of distinct output strings is distinguishable (see Theorem 3). These results are valid in a probabilistic sense, i.e. they hold with probability 
one with respect to the invariant probability measure for the given IFS.

The property of uniform left invertibility is of even greater interest for applications. We address such problem using a graph: paths on this graph are associated to orbits of the system. The main result about uniform left invertibility is Theorem 5, that gives necessary and sufficient conditions. Random Iteration Algorithm (RIA [8]) is also useful to study uniform left invertibility. This consists simply in choosing randomly input sequence linked to given probability distribution functions, and generating the corresponding orbits of the systems. A recent result in dynamical system theory (Theorem 2) tells us that the asymptotic probability of belonging to a given set in the state space is linked to the measure of the set (for the probability measure which is invariant for the IFS). Moreover such number can be computed by the RIA as limiting behavior. Finally, we illustrate our approach on examples.

The paper is organized as follows. The section 2 is devoted to the background knowledge, the section 3 to the basic setting of the paper, the definition of simple, uniform, and almost every distinguishability and invertibility. The section 4 shows principal results about almost every left invertibility and almost every uniform left invertibility: its principal result, Theorem 3, gives necessary and sufficient conditions for the almost every left invertibility. In section 5 the construction of invertibility graph is illustrated and a necessary and sufficient condition for left invertibility and uniform left invertibility is proved under some weak additional hypothesis. Section 6 contains examples about the difference between simple and uniform distinguishability and about the application of the method described in section 5 and 6. Section 7 shows conclusions and future perspectives.

\section{BACKGROUND}

Definition 1: Let $(\mathbb{X}, d)$ be a complete metric space. A map $F: \mathbb{X} \rightarrow \mathbb{X}$ is contractive if $\exists c \in \mathbb{R}, 0<c<1$ such that $d(F(x), F(y)) \leq c d(x, y)$ for all $x, y \in \mathbb{X} . \diamond$

Definition 2: An Iterated Function System with probabilities is a collection

$$
\left\{\mathbb{X}, F_{1}, \ldots, F_{n}, p_{1}, \ldots, p_{n}\right\},
$$

where where $(\mathbb{X}, d)$ is a metric space, $F_{i}: \mathbb{X} \rightarrow \mathbb{X}$ for $i=1, \ldots, n$, and $p_{i} \in \mathbb{R}$ such that $\sum_{i=0}^{n} p_{i}=1,0<p_{i}<1$, for $i=1, \ldots, n$. When the $p_{i}$ 's are not specified we refer to $\left\{\mathbb{X}, F_{1}, \ldots, F_{n}\right\}$ simply as an IFS. $\diamond$

We refer to [1], [11] for general theory of Iterated Function Systems (also called Iterated Function Schemes). In what follows we use $\sigma=\left\{\sigma_{i}\right\}_{i=1}^{\infty}$ to indicate a sequence of indices in $\{1, \ldots, n\}$. Moreover, for every $C \subset\{1, \ldots, n\}$ we indicate by $\Sigma_{C}$ the set of all sequence in $C$, and we define $\Sigma=\Sigma_{\{1, \ldots, n\}}$.

Definition 3: An orbit for the IFS (1) is a sequence $\{x(k)\}_{k=0}^{\infty}=\left\{x(k)_{x(0), \sigma}\right\}_{k=0}^{\infty} \subset \mathbb{X}$ given by the choice of an initial condition $x(0) \in \mathbb{X}$ and a sequence $\sigma \in \Sigma$, according to the following rule: $x(k+1)=F_{\sigma_{k}}(x(k)) . \diamond$
We now define, in a standard way, a measure on $\{1, \ldots, n\}^{\mathbb{N}}$.

Definition 4: For $i_{1}, \ldots, i_{r} \in \mathbb{N}, j_{1}, \ldots, j_{r} \in C$, the cylindrical subsets $v_{i_{1}, \ldots, i_{r}}^{j_{1}, \ldots, j_{r}}$ of $\Sigma_{C}$, is the set of strings defined by:

$$
\sigma \in v_{i_{1}, \ldots, i_{r}}^{j_{1}, \ldots, j_{r}} \Leftrightarrow \sigma_{k}=\left\{\begin{array}{c}
j_{1} \text { for } k=i_{1} \\
\ldots \\
j_{r} \text { for } k=i_{r}
\end{array} \diamond\right.
$$

A cylindrical subset $v_{i_{1}, \ldots, i_{r}}^{j_{1}, \ldots, j_{r}}$ is the set of all strings that on the $i_{k}-t h$ component assume the value $j_{k}$, for $k=1, \ldots, r$. The collection of all cylindrical subsets of $\Sigma$ generates a $\sigma$-algebra $\mathscr{B}$ on $\Sigma$. On these subsets we define the measure $\mu$ by

$$
\mu\left[v_{i_{1}, \ldots, i_{r}}^{j_{1}, \ldots, j_{r}}\right]=p_{j_{1}} \cdot \ldots \cdot p_{j_{r}} .
$$

This essentially means that the probability of the choice of the map $F_{i}$ is $p_{i}$ independently of the time. The equality (2) uniquely defines a probability measure on the entire $\sigma$-algebra $\mathscr{B}$ denoted by the same symbol $\mu$ (see [13]).

Definition 5: [3] A set $\mathscr{A}_{C} \subset \mathbb{X}$ is an attractor for the IFS (1) with respect to the index set $C$ if for all initial condition $x(0) \in \mathbb{X}$ and $\sigma \in \Sigma_{C}$ the orbit $\left\{x(k)_{x(0), \sigma}\right\}$ of the IFS (1) tends to $\mathscr{A}_{C}$ :

$$
\lim _{k \rightarrow \infty} d\left(x(k), \mathscr{A}_{C}\right)=0,
$$

where $d\left(x(k), \mathscr{A}_{C}\right)=\inf _{a \in \mathscr{A}_{C}} d(x(k), a) \cdot \diamond$

The orbit "is forced" to approach the attractor: we have then asymptotic information about orbits.

Definition 6: A set $\mathscr{I}_{C} \subset \mathbb{X}$ is an invariant set for the IFS (1) with respect to the index set $C$ if

$$
\mathscr{I}_{C}=\bigcup_{i \in C} F_{i}\left(\mathscr{I}_{C}\right) \cdot \diamond
$$

Note that, if $\mathscr{I}_{C}$ is an invariant set, given any initial condition $x(0)$ in $\mathscr{I}_{C}$, every possible orbit of the IFS (1) with indexes in $C$ is contained in $\mathscr{I}_{C}$. The next result shows that attractors and invariant sets exist for contractive IFS and are compact for all input sets $C$.

Theorem 1: [1][11][3] Let the IFS with probabilities (1) be contractive and let $C \subset\{1, \ldots, n\}$ be given. Then, for every $\sigma \in \Sigma_{C}$ the limit

$$
\phi(\sigma)=\lim _{k \rightarrow \infty} F_{\sigma_{1}} \circ \ldots \circ F_{\sigma_{k}}(x)
$$

exists for every $x \in \mathbb{X}$ and is independent of $x$. The set $\phi\left(\Sigma_{C}\right)=\mathscr{A}_{C}$ is the unique compact attractor and invariant set with respect to $\left\{F_{1}, \ldots, F_{n}\right\}$. Moreover, for all initial condition $x(0)$ and $\mu$-almost every $\sigma \in \Sigma_{C}$, the set $\{x(k)\}_{k \in \mathbb{N}} \cap \mathscr{A}_{C}$ is dense in $\mathscr{A}_{C} . \diamond$

Proposition 1: [1] Let $C_{1}, C_{2} \subseteq\{1, \ldots, n\}$ such that $C_{1} \subseteq$ $C_{2}$. Then $\mathscr{A}_{C_{1}} \subseteq \mathscr{A}_{C_{2}} \diamond$.

The attractor $\mathscr{A}_{C}$ in the Theorem 1 is easily algorithmic computable with the so called Random Iteration Algorithm ([8]). Let us briefly illustrate how the Random Iteration Algorithm proceeds. An initial point $x_{0} \in \mathbb{X}$ is chosen. One of the transformations is selected "at random" from the set $\left\{F_{1}, \ldots, F_{n}\right\}$, but the probability that each $F_{i}$ is selected is $p_{i}$ for $i=1, \ldots, n$. The selected transformation is applied 
to produce a new point $x_{1} \in \mathbb{X}$. Again a transformation is selected using associated probabilities, in the same manner, independently from the previous choice, and applied to $x_{1}$ to produce a new point $x_{2}$, and so on. The application of the Random Iteration Algorithm with any initial condition let the orbit tend to the attractor (see [1][8]). Moreover, the Random Iteration Algorithm has further properties, due to ergodic properties of the dynamics on the attractor of an IFS:

Theorem 2 (Elton): [10]

Let $\left\{\mathbb{X}, F_{1}, \ldots, F_{n}, p_{1}, \ldots, p_{n}\right\}$ be an IFS, and indicate with $\left\{x_{k}\right\}_{k=0}^{\infty}$ a generic orbit of the IFS. Let $B$ Borel subset of $\mathbb{X}$ with boundary of zero Lebesgue measure. Let $\mathscr{N}(B, k)$ be the number of points in $\left\{x_{0}, \ldots, x_{k}\right\} \cap B$. Then, for all initial conditions $x_{0}$ and $\mu$-almost every $\sigma \in \Sigma$ the limit

$$
L_{B}=\lim _{k \rightarrow \infty}\left\{\frac{\mathscr{N}(B, k)}{k+1}\right\}
$$

exists and assumes a common value. $\diamond$

Remark 1: Both the Theorem 1,2 have a "practical" interpretation: running the Random Iteration Algorithm these results holds "almost surely", namely with probability one with respect to the measure $\mu$. This is of course desirable because it allows the help of computers. $\diamond$

There is another way of looking at IFS, or equivalently at their attractors, that make the IFS "equivalent", in a precisely specified sense, to a space of strings, namely the code space:

Definition 7: The code space $\Sigma$ associated with the IFS (1), is the space of all sequences on $n$ symbols. An address of a point $a \in \mathscr{A}_{\{1, \ldots, n\}}$ is any member of the set $\phi^{-1}(a)=\{\sigma \in$ $\Sigma: \phi(\sigma)=a\}$. The attractor is said to be totally disconnected if each point possesses a unique address. $\diamond$

Proposition 2: [1] The attractor $\mathscr{A}=\mathscr{A}_{\{1, \ldots, n\}}$ is totally disconnected if and only if $F_{i}(\mathscr{A}) \cap F_{j}(\mathscr{A})=\emptyset \quad \forall i \neq j . \diamond$

\section{BASIC SETTING}

In this paper we consider discrete-time, time-invariant, non-linear systems of the form

$$
\left\{\begin{array}{l}
x(k+1)=f(x(k), u(k))=f_{u(k)}(x(k)) \\
y(k)=q(x(k))
\end{array}\right.
$$

where $x(k) \in \mathbb{R}^{d}$ is the state, $y(k) \in \mathscr{Y}$ is the output, and $u(k) \in \mathscr{U}$ is the input. We assume that $\mathscr{Y}$ is a discrete set. The map $q: \mathbb{R}^{d} \rightarrow \mathscr{Y}$ is induced by a partition $\mathscr{P}=\bigcup_{i=1}^{M} P_{i}$ of $\mathbb{R}^{d}$ through $q:\left(x \in P_{i}\right) \mapsto i$ and will be referred to as the output quantizer. We admit infinite partitions, i.e. $M \in \mathbb{N} \cup+\infty$, but we assume that $\mathscr{U}$ is a finite set of cardinality $m$. With no loss of generality (modulo redefining the dynamics $f(\cdot, \cdot)$ and the function $q$ ), we will assume $\mathscr{U}=\{1, \ldots, m\}$ and $\mathscr{Y}=\{1, \ldots, M\}$. We further assume that the system dynamics are contractive, in the sense of the following definition:

Hyp $1: f_{u}: \mathbb{R}^{d} \rightarrow \mathbb{R}^{d}, \quad u=1, \ldots, m$, is a contraction. $\diamond$

We suppose that to each $i \in\{1, \ldots, m\}$ it is associated a real number $p_{i}$ with $0<p_{i}<1, \sum_{i=1}^{m} p_{i}=1$ that is interpreted as the probability of the event $\{u(k)=i\}$.
If $x_{0}$ is an initial condition and $\left(u_{1}, \ldots, u_{k_{2}}\right)$ a sequence of inputs, we let $f_{k_{1}}^{k_{2}}\left(x_{0}, u_{1}, \ldots, u_{k_{2}}\right)$ denote the sequence of outputs $\left(y_{k_{1}}, \ldots, y_{k_{2}}\right)$ generated by the system (5) with initial condition $x_{0}$ and input string $\left(u_{1}, \ldots, u_{k_{2}}\right)$.

Definition 8: A pair of inputs strings $\left\{u_{i}\right\}_{i \in \mathbb{N}},\left\{u_{i}^{\prime}\right\}_{i \in \mathbb{N}}$ is distinguishable if $\forall x_{0}, x_{0}^{\prime} \in \mathbb{R}^{d} \exists k=k\left(x_{0}, x_{0}^{\prime},\left\{u_{i}\right\},\left\{u_{i}^{\prime}\right\}\right) \in \mathbb{N}$ such that

$$
f_{0}^{k}\left(x_{0}, u_{1}, \ldots, u_{k}\right) \neq f_{0}^{k}\left(x_{0}^{\prime}, u_{1}^{\prime}, \ldots, u_{k}^{\prime}\right) . \diamond
$$

Definition 9: A pair of input strings $\left\{u_{i}\right\}_{i \in \mathbb{N}},\left\{u_{i}^{\prime}\right\}_{i \in \mathbb{N}}$ is uniformly distinguishable if for every compact set $K \in \mathbb{R}^{d} \times$ $\mathbb{R}^{d}$ there exists $k=k\left(\left\{u_{i}\right\},\left\{u_{i}^{\prime}\right\}\right) \in \mathbb{N}, l=l(K)$ such that $\forall\left(x_{0}, x_{0}^{\prime}\right) \in K$ and $\forall m>l$ the following holds:

$u_{m} \neq u_{m}^{\prime} \Rightarrow f_{m}^{m+k}\left(x_{0}, u_{1}, \ldots, u_{m+k}\right) \neq f_{m}^{m+k}\left(x_{0}^{\prime}, u_{1}^{\prime}, \ldots, u_{m+k}^{\prime}\right)$. In this case, we say that the strings are uniformly distinguishable in $k$ steps (or with invertibility time $k$ ) and after time $l$ (or with waiting time $l$ ). $\diamond$

Definition 10: A system of type (5) is left invertible if the following holds:

$\checkmark$ For every $u, u^{\prime} \in \mathscr{U}^{\mathbb{N}}$, there exists $l\left(u, u^{\prime}\right) \in \mathbb{N}$ such that, if $u_{i} \neq u_{i}^{\prime}$ with $i>l$, then the pair $u, u^{\prime}$ is distinguishable. $\nabla$

Definition 11: A system of type (5) is $\mu$-almost everywhere left invertible if the relation $\checkmark$ in Definition 10 is valid for $\mu$-almost every pair of different input strings, where $\mu$ is the measure defined in (2). $\diamond$

Definition 12: A system of type (5) is uniformly left invertible in $k$ steps if, for initial conditions in a compact set $K \subset \mathbb{R}^{d} \times \mathbb{R}^{d}$, every pair of distinct input sequences is uniformly distinguishable in $k$ steps after a finite time $l$, where $k$ is constant and $l$ depends only on $K$.

A system of type (5) is $\mu$-almost everywhere uniformly left invertible in $k$ steps if, for initial conditions in a compact set $K \subset \mathbb{R}^{d} \times \mathbb{R}^{d}, \mu$-almost every pair of distinct input sequences is uniformly distinguishable in $k$ steps after a finite time $l$, where $k$ is constant and $l$ depends only on $K(\mu$ is the measure defined in $(2)) . \diamond$

So, for a left invertible system, it is possible to recover infinite input strings observing the corresponding infinite output strings. For a uniform left invertible system, it is possible to recover the input string until the instant $m$ observing the output string until the instant $m+k$, and to obtain an algorithm to reconstruct the input symbol used at time $m>l$ by processing the output symbols from time $m$ to $m+k$.

\section{ATTRACTORS AND LEFT INVERTIBILITY}

In this section we introduce a technique that links the invertibility problem to the theory of Iterated Function Systems and the properties of their attractors. Define

$$
Q=\bigcup_{y \in \mathscr{Y}}\left\{q^{-1}(y) \times q^{-1}(y)\right\} \subset \mathbb{R}^{2 d}
$$

i.e. the union of the preimages of two identical output symbols. In other words, $Q$ contains all pairs of states that are in the same element of the partition $\mathscr{P}$. 
To address left invertibility, we are interested in studying the following system on $\mathbb{R}^{2 d}$ :

$$
X(k+1)=F_{U(k)}(X(k))=\left[\begin{array}{c}
f\left(x_{1}(k), u(k)\right) \\
f\left(x_{2}(k), u^{\prime}(k)\right)
\end{array}\right]
$$

where $X(k)=\left[\begin{array}{c}x_{1}(k) \\ x_{2}(k)\end{array}\right] ; \quad U(k)=\left(u(k), u^{\prime}(k)\right)$;

If it is possible to find an initial state in $Q$ and an appropriate choice of the strings $\left\{u_{k}\right\},\left\{u_{k}^{\prime}\right\}$ such that the orbit of (6) remains in $Q$, it means that the two strings of inputs give rise to the same output for the system (5). Conditions ensuring that the state is outside $Q$ will be seeked to guarantee left invertibility.

Definition 13: The IFS associated to the system (6) is

$$
\left\{K ; F_{(1,1)}, F_{(1,2)}, \ldots, F_{(m, m)} ; P_{(1,1)}, P_{(1,2)} \ldots, P_{(m, m)}\right\},
$$

where $K$ is a compact subset of $\mathbb{R}^{2 d}$, and $P_{i, j}=p_{i} p_{j} . \diamond$

Definition 14: For any $C \subset \mathscr{U} \times \mathscr{U}$ it is denoted by $\Sigma_{C}$ the set of all sequences $\sigma=\left\{\sigma_{i}\right\}_{i=0}^{\infty}$ such that $\sigma_{i} \in C$ for every $i \in \mathbb{N}$. Moreover we define $n=m^{2}$ and $\Sigma=\Sigma_{\mathscr{U} \times \mathscr{U}}$ to be the code space on $n$ elements. $\diamond$

Thanks to the Theorem 1, given a system of type (5) and a subset of input symbols $C$ for the corresponding system of type (6), it is possible to describe a set $\mathscr{A}_{C}$ that is both an attractor and an invariant set.

Note that the attractor associated to a single $U \in \mathscr{U} \times$ $\mathscr{U}$, indicated by $X_{U}$, by Contraction Theorem (see [12]), is a unique fixed point, and it can be approximated iterating the map $F_{U}$. For every $U \in \mathscr{U} \times \mathscr{U}$, for all $X \in \mathbb{R}^{2 d} X_{U}=$ $\lim _{k \rightarrow \infty} F_{U}^{k}(X)$. The relative position of these fixed points with respect to $Q$ is sufficient to conclude about the $\mu$-almost every left invertibility. Let $\Delta$ denote the diagonal of $\mathscr{U} \times \mathscr{U}$, i.e. $\Delta=\{(1,1),(2,2), \ldots,(m, m)\}$.

Theorem 3: If there exists $U \notin \Delta$ such that $X_{U} \subset Q$, then the system (5) is not left invertible. If every $X_{U}, U \notin \Delta$ is not in $\bar{Q}$, the closure of $Q$, the system (5) is $\mu$-almost everywhere left invertible. $\diamond$

Proof: Suppose that there exists $U \notin \Delta$ such that $X_{U} \subset Q$. Select $X_{U}$ as initial condition and choose $\sigma$ to be the constant sequence $\sigma_{i}=U \forall i \in \mathbb{N}$. The resulting orbit is the constant orbit $X(i)=X_{U} \forall i \in \mathbb{N}$, and it is clearly contained in $Q$, so the system is not left invertible.

Suppose that $U \in \mathscr{U} \times \mathscr{U}, U \notin \Delta \Rightarrow X_{U} \notin \bar{Q}$. First observe that in this hypothesis no attractors $\mathscr{A}_{C}, C \not \subset \Delta$ are included in $\bar{Q}$, because of the Proposition 1: indeed every attractor $\mathscr{A}_{C}, C \not \subset \Delta$ must contain a $X_{U}, U \notin \Delta$.

So Theorem 1 assures that for $\mu$-almost every couple of input strings the trajectory is dense in $\mathscr{A}$. So, if $\mathscr{A}$ has a point $p$ not in $\bar{Q}$, the generic trajectory contains points arbitrarily close to $p$ and so contains points that are not included in $Q$. This proves the $\mu$-almost everywhere left invertibility result. $\diamond$

\section{INVERTIBILITY GRAPH AND LEFT INVERTIBILITY}

We now introduce a graph, whose properties are linked to uniform left invertibility.
Definition 15: The graph $G_{k}$ of depth $k$ associated to the attractor $\mathscr{A}$ is given by:

- The set of vertices $V=\left\{\mathscr{A}_{\sigma_{1} \ldots \sigma_{k}}=F_{\sigma_{k}} \circ \ldots \circ F_{\sigma_{1}}(\mathscr{A}): \sigma_{i} \in\right.$ $\Sigma\}$

- There is an edge from $\mathscr{A}_{\sigma_{1} \ldots \sigma_{k}}$ to $\mathscr{A}_{\omega_{1} \ldots \omega_{k}}$ if and only if $\sigma_{i+1}=\omega_{i}$, for $i=1, \ldots, k-1$. In this case we say that the edge is induced by the input $\omega_{k} . \diamond$

Remark 2: It follows from the definition of $G_{k}$ that

1) If there is an edge between $\mathscr{A}_{\sigma_{1} \ldots \sigma_{k}}$ and $\mathscr{A}_{\omega_{1} \ldots \omega_{k}}$, then there exists $U \in \mathscr{U} \times \mathscr{U}$ such that $F_{U}\left(\mathscr{A}_{\sigma_{1} \ldots \sigma_{k}}\right) \subset$ $\mathscr{A}_{\omega_{1} \ldots \omega_{k}}$.

2) $\bigcup_{\sigma_{i} \in \Sigma} \mathscr{A}_{\sigma_{1} \ldots \sigma_{k}}=\mathscr{A}$ : i.e. the union of vertexes of $G_{k}$, considered as sets, is the whole attractor.

3) If the attractor $\mathscr{A}$ is totally disconnected, the vertices of $G_{k}$ provide a partition of the attractor. $\diamond$

Definition 16: Consider the graph $G_{k}$, and collapse to a single vertex, denoted by $\mathscr{A}_{I}$, all vertices $\mathscr{A}_{\sigma_{1} \ldots \sigma_{k}}$ such that $\mathscr{A}_{\sigma_{1} \ldots \sigma_{k}} \cap\left\{\mathbb{R}^{2 d} \backslash Q\right\} \neq \emptyset$. Moreover every edge from $\mathscr{A}_{I}$ is deleted. This new graph is called internal invertibility graph, and denoted with $I G_{k}$. The set of vertices of $I G_{k}$ is denoted by $V_{I G_{k}}$.

Now consider the graph $G_{k}$, and collapse to a single vertex, denoted by $\mathscr{A}_{E}$, all vertices $\mathscr{A}_{\sigma_{1} \ldots \sigma_{k}}$ such that $\mathscr{A}_{\sigma_{1} \ldots \sigma_{k}} \cap$ $Q=\emptyset$. This new graph is called external invertibility graph, and denoted by $E G_{k}$. The set of vertices of $E G_{k}$ is denoted by $V_{E G_{k}}$. In the following we use the symbols $V_{I G_{k}}, V_{E G_{k}}$ to denote the vertices of the graphs as well as the set of points that they represent. $\diamond$

Definition 17: A path $\left\{V_{i}\right\}_{i \in \mathbb{N}}$ on $E G_{k}$ or $I G_{k}$ is called proper if for every $l \in \mathbb{N}$ there exists an input $U_{i} \notin \Delta$ with $i>l$, that induces the edge $V_{i} \rightarrow V_{i+1}$.

A finite path $\left\{V_{i}\right\}_{i=0}^{m}$ on $E G_{k}$ or $I G_{k}$ is called finite proper path if the first edge is induced by an input not in $\Delta$. $\diamond$

Proposition 3: There exists an orbit of the system (6) included in the set of vertices of $V_{I G_{k}} \backslash \mathscr{A}_{I}$, if and only if there exists an infinite path in $I G_{k}$.

Proof: If there is an orbit $\left\{X_{i}\right\}=\left\{\sigma_{i} \circ \ldots \circ \sigma_{1}\left(X_{0}\right)\right\}_{i=1}^{\infty}$ included in $V_{I G_{k}} \backslash \mathscr{A}_{I}$, then we can construct an infinite path on $I G_{k}$ by associating to each $X_{i}, i \geq k$ the vertex $\mathscr{A}_{\sigma_{i-k}, \ldots, \sigma_{i-1}}$ such that $X_{i} \in \mathscr{A}_{\sigma_{i-k}, \ldots, \sigma_{i-1}}$. It is really a path (of infinite length) on the graph $I G_{k}$ thanks to the Remark 2 , and never touches $\mathscr{A}_{I}$.

Conversely, if there is an infinite path $\left\{V_{1}, V_{2}, \ldots\right\}$ in $I G_{k}$, first note that it cannot touch $\mathscr{A}_{I}$ because there is no edge starting from $\mathscr{A}_{I}$. Then, thanks to the first part of Remark 2, it is possible to exhibit an orbit included in $V_{I G_{k}} \backslash \mathscr{A}_{I}$. Indeed if any $X_{1} \in V_{1}$ is chosen, then there exists an $U_{1} \in \mathscr{U} \times \mathscr{U}$ such that $X_{2}=F_{U}\left(X_{1}\right) \in V_{2}$; there exists $U_{2} \in \mathscr{U} \times \mathscr{U}$ such that $X_{3}=F_{U}\left(X_{2}\right) \in V_{3}$. Continuing in this way for every $i \in \mathbb{N}$ it is found an $U_{i-1} \in \mathscr{U} \times \mathscr{U}$ such that $X_{i}=F_{U}\left(X_{i-1}\right) \in$ $V_{i}$. This procedure gives rise to an orbit of the system (6) included in $V_{I G_{k}} \backslash \mathscr{A}_{I} . \diamond$

Proposition 4: Fix $i \in \mathbb{N}$. A sufficient condition for the uniform left invertibility of the system (6) is the absence of finite proper paths of arbitrary length in $E G_{i}$. 
Proof: Suppose that the system (6) is not uniformly left invertible. Then, for every $j \in \mathbb{N}$ there exists an orbit $\left\{X_{p}\right\}=$ $\left\{\sigma_{p} \circ \ldots \circ \sigma_{1}\left(X_{0}\right)\right\}_{p=1}^{\infty}$ such that $X_{1}=F_{U}\left(X_{0}\right)$ with $U \notin \Delta$ and $\left\{X_{p}\right\}_{p=0}^{j} \subset Q \cap \mathscr{A}$. Then we can construct a path on $E G_{i}$ by associating at each $X_{p}, p \geq i$ the vertex $\mathscr{A}_{\sigma_{p-i}, \ldots, \sigma_{p-1}}$ such that $X_{p} \in \mathscr{A}_{\sigma_{p-i}, \ldots, \sigma_{p-1}}$. It is really a proper path (because $X_{1}=F_{U}\left(X_{0}\right)$ with $\left.U \notin \Delta\right)$ on the graph $E G_{i}$ thanks to the Remark 2, and for every $p \leq j V_{p} \neq \mathscr{A}_{E}$, because $\mathscr{A}_{E} \cap$ $Q=\emptyset$ and $\left\{X_{p}\right\}_{p=0}^{j} \subset Q$. It remains to note that, since this construction can be made for every $j \in \mathbb{N}$, proper paths of arbitrary length are present in $E G_{i}$. $\diamond$

Proposition 5: Suppose that no point of $\mathscr{A}$ belongs to the boundary $\partial Q$ of $Q$, or equivalently that $\inf _{a \in \mathscr{A}} d(a, \partial Q)>0$. Then there exists a $k \in \mathbb{N}$ such that $V_{I G_{k}} \backslash \mathscr{A}_{I}=V_{I G_{k}} \cap Q$. This in turn implies that $I G_{k}=E G_{k}$

Proof: We first show that $\mathscr{A} \cap \partial Q=\emptyset$ if and only if $\inf _{a \in \mathscr{A}} d(a, \partial Q)>0$.

Suppose that $\mathscr{A} \cap \partial Q=\emptyset$. If $\inf _{a \in \mathscr{A}} d(a, \partial Q)=0$, then choose a sequence $\left\{a_{k}\right\} \subset \mathscr{A}$ such that $\lim _{k \rightarrow \infty} a_{k} \searrow 0$. Since $\mathscr{A}$ is compact there is an accumulation point $a \in \mathscr{A}$ for $\left\{a_{k}\right\}$. Then it is immediate to see that $d(a, \partial Q)$ must be zero. So $a$ should belong to $\partial Q$ because $\partial Q$ is a closed set. This is impossible because we supposed that $\mathscr{A} \cap \partial Q=\emptyset$. So it must be $\inf _{a \in \mathscr{A}} d(a, \partial Q)>0$. Conversely if $\inf _{a \in \mathscr{A}} d(a, \partial Q)>0$, then $\mathscr{A} \cap \partial Q=\emptyset$.

So, assume now that $c=\inf _{a \in \mathscr{A}} d(a, \partial Q)>0$. Choose $k$ such that every set $V_{I G_{k}}^{i}$ has a diameter $\delta_{i}<c$. Then $V_{I G_{k}} \backslash$ $\mathscr{A}_{I}=V_{I G_{k}} \cap Q$ because no $V_{I G_{k}}$ can intersect $\partial Q . \diamond$

Theorem 4: Suppose that $\mathscr{A} \cap \partial Q=\emptyset$. Then the following conditions are equivalent:

1) The system (5) is left invertible;

2) $I G_{\bar{k}}$ does not contain a proper path, where $\bar{k}$ is such that $V_{I G_{\bar{k}}} \backslash \mathscr{A}_{I}=V_{I G_{\bar{k}}} \cap Q$;

Proof: Suppose that $I G_{\bar{k}}$ contains a proper path $\left\{V_{i}\right\}_{i \in \mathbb{N}}$. By Proposition 3 there exists an orbit $\left\{X_{i}\right\}_{i \in \mathbb{N}}$, where $X_{i} \in V_{i}$. Note that $X_{i} \notin \mathscr{A}_{I}$ because no edges start from $\mathscr{A}_{I}$. The orbit is included in $Q$ because $X_{i} \in V_{I G_{\bar{k}}} \backslash \mathscr{A}_{I}=V_{I G_{\bar{k}}} \cap Q$. Moreover $U \notin \Delta$ for an infinite number of times, because the path is proper. This contradicts left invertibility.

Viceversa, suppose that the system (5) is not left invertible. Then there exists an orbit $\left\{X_{i}\right\}_{i \in \mathbb{N}} \subset Q$, such that $X_{i}=F_{U}\left(X_{i-1}\right)$ and $U \notin \Delta$ for an infinite number of $i \in \mathbb{N}$. This orbit, induces, in the same way as in the proof of Proposition 3, a proper path in $I G_{\bar{k}} . \diamond$

Theorem 5: Suppose that $\mathscr{A} \cap \partial Q=\emptyset$. Then the following conditions are equivalent:

1) The system (5) is uniformly left invertible;

2) $I G_{\bar{k}}$ does not contain finite proper path of arbitrary lengths, where $\bar{k}$ is such that $V_{I G_{\bar{k}}} \backslash \mathscr{A}_{I}=V_{I G_{\bar{k}}} \cap Q$;

Proof: Suppose that $I G_{\bar{k}}$ contains finite proper paths of arbitrary length $\left\{V_{i}^{\alpha}\right\}_{i=0}^{m^{\alpha}}$. By Proposition 3 there exists an orbit $\left\{X_{i}^{\alpha}\right\}_{i \in \mathbb{N}}$, where $X_{i}^{\alpha} \in V_{i}^{\alpha}$. The orbit is included in $Q$ because $X_{i}^{\alpha} \in V_{I G_{\bar{k}}} \backslash \mathscr{A}_{I}=V_{I G_{\bar{k}}} \cap Q$. Moreover $X_{1}^{\alpha}=F_{U^{\alpha}}\left(X_{0}^{\alpha}\right)$, with $U^{\alpha} \notin \Delta$. This contradicts uniform left invertibility. The inverse implication is true by Proposition $4 . \diamond$

\section{EXAMPLES}

Consider the system

$$
\left\{\begin{array}{l}
x(k+1)=J_{d}^{\alpha}(a) x(k)+\mathbf{1}_{d} u(k) \\
y(k)=\left\lfloor\pi_{1} x(k)\right\rfloor
\end{array}\right.
$$

Where $J_{d}^{\alpha}(a)$ is the matrix of dimension $d$ with $a$ 's on the principal diagonal and $\alpha$ in the upper one, with $x(k) \in \mathbb{R}^{d}$, $y(k) \in \mathbb{Z}, \mathbf{1}_{d}=(1,1, \ldots, 1)^{T}, u(k) \in\left\{u_{1}, \ldots, u_{m}\right\} \subset \mathbb{R}$, and $\lfloor\cdot\rfloor$ is the floor function. We examine a situation in which all inputs are equally probable, i.e. we know no information about recurrence of inputs: $\forall i=1, \ldots, m \quad p_{i}=\frac{1}{m}$. Define $U_{(i, j)}=\left(\mathbf{1} u_{i}, \mathbf{1} u_{j}\right)^{T}, S_{i}=[0, i), S=\left(S_{3} \times S_{3} \times S_{3} \times S_{3} \times S_{3}\right)^{2}$, and $M=\left(\begin{array}{cc}J_{5}^{1 / 2}\left(\frac{1}{10}\right) & 0 \\ 0 & J_{5}^{1 / 2}\left(\frac{1}{10}\right)\end{array}\right)$.

Example 1: Consider the system (8), with $d=5, a=$ $1 / 10, \alpha=1 / 2$ and $u \in\{0,1 / 20,1\}$. Then we have, for example, $X_{(0,1 / 20)} \in Q\left(X_{(0,1 / 20)}\right.$ can be computed iterating the transformation $\left.x \mapsto M x+U_{(0,1 / 20)}\right)$, so the system is not left invertible by Theorem 3 .

Consider instead the system (8), with $\mathscr{U} \in\{0,1\}$. Then $X_{(0,1)} \notin Q$ and $X_{(1,0)} \notin Q$. By Theorem 3 the system is $\mu$-almost everywhere left invertible.

We are going to show that the system is indeed uniformly left invertible. Direct calculations show that

$$
\begin{gathered}
\bigcup_{(i, j) \in\{0,1\}^{2}} M(S)+U_{(i, j)} \subset S ; \\
U_{(i, j)} \neq U_{\left(i^{\prime}, j^{\prime}\right)} \Rightarrow M(S)+U_{(i, j)} \bigcap M(S)+U_{\left(i^{\prime}, j^{\prime}\right)}=\emptyset ; \\
(i, j) \notin \Delta \Rightarrow M(S \cap Q)+U_{(i, j)} \bigcap Q=\emptyset .
\end{gathered}
$$

Equation (9) implies by Theorem 1 that $S$ contains the attractor, equation (10) implies by theorem 2 that the attractor is totally disconnected, and equation (11) implies by Theorem ?? that the system is uniformly left invertible in 1 step. $\diamond$

Finally, we give another example in dimension 1, drawing the attractor of the system and the invertibility graph.

Example 2: Consider a linear system with

$$
d=1, a=1 / 5, \mathscr{U}=\{0.2,0.9\}
$$

System (12) is contractive because $a<1$. The attractor of the system, in Figure 2, has been drawn with Random Iteration Algorithm, and it is totally disconnected. Indeed calculations show that it is included in the square $\mathscr{S}=$ $[0.2,1.2] \times[0.2,1.2]$, and that

$$
\left\{1 / 5 \cdot \mathscr{S}+\left(u_{1}, u_{2}\right)\right\} \bigcap\left\{1 / 5 \cdot \mathscr{S}+\left(u_{3}, u_{4}\right)\right\}=\emptyset
$$

if $\left(u_{1}, u_{2}\right) \neq\left(u_{3}, u_{4}\right) \in \mathscr{U} \times \mathscr{U}$. This suffices, by Proposition 2 , to conclude that the attractor of system (12) is totally disconnected. To our purpose we can divide the attractor in 16 parts (indicated by a circle around them), each one being represented by an address of two symbols in the alphabet $\mathscr{U} \times \mathscr{U}$. Then direct calculations shows that $I G_{2}$ and $E G_{2}$ coincide, and that the resulting graph is that one of figure 2, where we write $U_{i} U_{j}$ instead of $\mathscr{A}_{U_{i} U_{j}}$, 


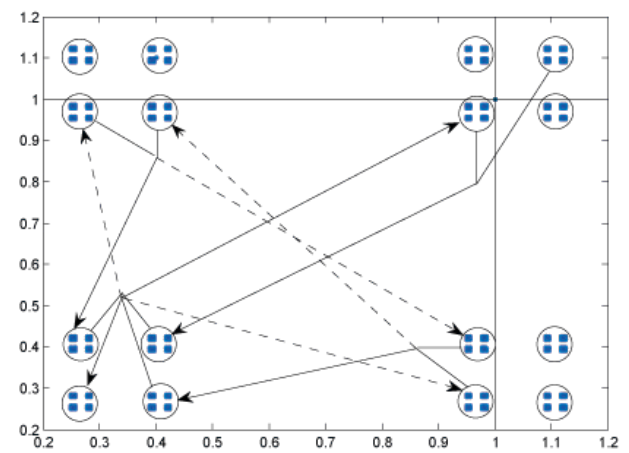

Fig. 1. Attractor of system (12)

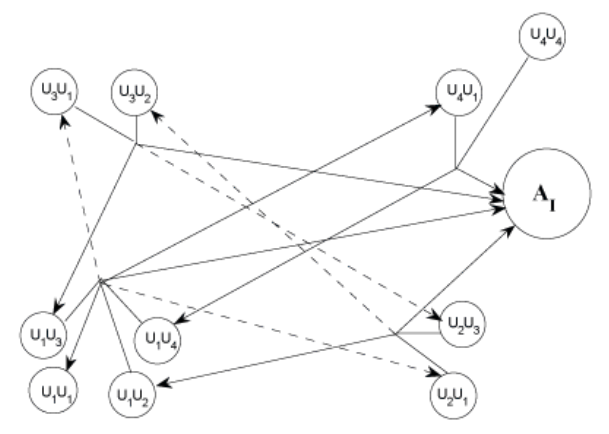

Fig. 2. $I G_{2}$ of system (12)

with $U_{1}=(0.2,0.2), U_{2}=(0.9,0.2), U_{3}=(0.2,0.9)$, and $U_{4}=(0.9,0.9)$. Moreover dashed edges are drawn when they start a proper path on the graph. Clearly there exists infinite proper paths in $I G_{2}$ (for instance the periodic path obtained repeating the edges $U_{2} U_{3}$ and $U_{3} U_{2}$ ), so system (12) is neither left invertible nor uniformly left invertible. $\diamond$

\section{CONCLUSIONS}

In this paper we introduced a technique relating the theory of IFS to the left invertibility problem, for contractive dynamics. A necessary and sufficient condition for left invertibility with probability one with respect to input strings is given (Theorem 3), and necessary and sufficient conditions for left invertibility and uniform left invertibility are stated (Theorems 4, 5). In particular we showed that left invertibility of contractive systems depends only on the properties of a compact set (the attractor), that is easily algorithmically approximable. Many questions for future investigation are of interest: the possibility of extending the results to non-contractive dynamics and to other state spaces such as manifolds. Of particular interest is the possibility of building an algorithm starting from Theorems 4, 5 to check left invertibility.

\section{REFERENCES}

[1] BARNSLEY M., Fractals everywhere, Academic Press inc., (1993).
[2] BICCHI A., MARIGO A., PICCOLI B., On the reachability of quantized control sytems, IEEE Transactions on Automatic Control, 47, 4, (2002), 546-563.

[3] BOBYLEV N. A., EMEL'YANOV S. V., KOROVIN S. K., Attractor of discrete controlled systems in metric spaces, Computational Mathematics and modeling, 11, 4, (2000), 321-326.

[4] BROCKETT R. W., and MESAROVIC M. D., The reproducibility of multivariable control systems, J. Math. Anal. Appl., 11, (1965), 548-563.

[5] BROOMHEAD D. S., HUKE J. P., MULDOON M. R., STARK J., Iterated function system models of digital channels, Proceedings of the Royal Society of London, A-460, (2004), 3123-3142.

[6] CURRY R. E., Estimation and control with quantized measurements, Research Monograph, M.I.T. PRESS, (1970).

[7] DELCHAMPS D. F., Stabilizing a linear system with quantized state feedback, IEEE Transactions on Automatic Control, 35, 8, (1990), 916-924.

[8] DIACONIS P., FREEDMAN D., Iterated random functions, SIAM Rev., 41, (1999), 45-76.

[9] EDELMAYER A., BOKOR J., SZABÓ Z., SZIGETI F., Input reconstruction by means of system inversion: a geometric approach to fault detection and isolation in nonlinear systems, Int. J. Appl. Math. Comput. Sci., 14, 2, (2004), 189-199.

[10] ELTON J., An ergodic theorem for iterated maps, Journal of ergodic theory and dynamical systems, 7, (1987), 481-488.

[11] FALCONER K., Fractal geometry, mathematical foundations and applications, John Wiley and Sons, (2003).

[12] GRANAS A., DUGUNDJI J., Fixed Point Theory, Springer-Verlag, New York, (2003).

[13] HALMOS P. R., Measure Theory, Springer-Verlag, (1978).

[14] HUTCHINSON J. E., Fractals and self-similarity, Indiana University Math. J. 30, (1981), 713-747.

[15] INOUE E., USHIO T., Chaos communication using unknown input observer, Electronic and Comunication in Japan, Part 3, 84, 12, (2001).

[16] MASSEY J. L., SAIN M. K., Invertibility of linear time-invariant dynamical systems, IEEE Transactions on Automatic Control, AC-14, 2, (1969), 141-149.

[17] MASSEY J. L., SAIN M. K., Inverses of linear sequential circuits, IEEE Trans. Computers, C-17, (1968), 330-337.

[18] MORAN P. A. P., Additive functions of intervals and Hausdorff measure, Proc. Camb. Phil. Soc. 42, (1946), 15-23.

[19] MORSE A. S., WONHAM W. M., Status of noninteracting control, IEEE Trans. Automat. Control, 16, 6, (1971), 568-581.

[20] NIJMEINER H., Right-invertibility for a class of nonlinear control systems: A geometric approach, Systems and Control Letters, 7, 2, (1986), 125-132.

[21] PICASSO B., BICCHI A., On the stabilization of linear systems under assigned I/O quantization, IEEE Transactions on Automatic Control, 52, 10, (2007), 1994-2000.

[22] RADOSLAV S., Inverse problem of nonlinear system dynamics (book chapter), Analysis and optimization of systems, Springer, (2006).

[23] RESPONDEK W., Right and Left Invertibility of Nonlinear Control Systems, Nonlinear Controllability and Optimal Control, New York, (1990), 133-176.

[24] SACCO, P. L., SANDRI M., Input-Output dynamics as an iterated function systems, Working paper no. 12 of Economic Sciences Institute, University of Verona, (1993).

[25] SILVERMAN L. M., Inversion of multivariable linear systems, IEEE Trans. Automat. Control, 14, 3, (1969), 270-276.

[26] SONTAG E. D., Mathematical control theory: deterministic finite dimensional systems, Springer, New York, (1998).

[27] SZANIER M., SIDERIS A., Feedback control of quantized constrained systems with applications to neuromorphic controller design, IEEE Transactions on Automatic Control, 39, 7, (1994), 1497-1502.

[28] TATIKONDA S. C., MITTER S., Control under communication constraints, IEEE Transactions on Automatic Control, 49, 7, (2004), 1056-1068.

[29] VU L., LIBERZON D., Invertibility of switched linear systems, Proceedings of the 45th IEEE Conference on Decision and Control, (2006), 4081-4086. 